\title{
Dinámica de grupos precerámicos en el perfil costa-altiplano, norte de Chile*
}

LAUTARo NúÑ̃z A.**

\section{Introducción}

Este artículo intenta presentar diversos casos de desplazamientos de grupos precerámicos registrados en la faja central de la provincia de Tarapacá, en el extremo árido del norte de Chile. Para este efecto se han reunido evidencias arqueológicas más o menos sincrónicas, a través del estudio de un conjunto de sitios recientemente reconocido, y que representa diversas situaciones en relación al temprano manejo de los recursos naturales del área.

Con este objetivo se presentan las condiciones ecológicas que existieron en diversos microambientes ubicados entre la costa y el altiplano de la zona de estudio. Los casos seleccionados y presentados señalarían algunas categorías diferentes de explotación de los microambientes locales, los cuales habrían estimulado diversos circuitos de desplazamientos transhumánticos. En este sentido, los microambientes detectados se evalúan en relación a su estricta interdependencia con los asentamientos humanos. El equilibrio entre los recursos de un área afectada por el proceso de aridez y los campamentos ubicados en cada uno de los nichos ecológicos de una zona restringida, supone la existencia de un proceso de adaptación regional basado en un régimen de explotación multiecológica, por medio de circuitos transhumánticos.

\section{Marco teórico}

Un conjunto selecto de sitios precerámicos ubicados entre la costa y el altiplano del norte chileno $\left(19^{\circ}\right.$ a $27^{\circ}$ de latitud y $71^{\circ}$ a $69^{\circ}$ de longitud) presentan diferentes evidencias de acuerdo a la variedad ecológica de los nichos que ocupan en una de las áreas actuales más desérticas del mundo. El análisis de las condiciones ecológicas actuales permite hacer inferencias sobre el ambiente que debió existir entre los 4000 a 2000 años AC, debido a que el proceso de aridez local

\footnotetext{
* Artículo presentado al XLI Congreso Internacional de Americanistas, México, 1974. Sesión Especial "Sistemas ecológicos prehistóricos de los Andes"

** Universidad del Norte, Antofagasta, Chile.
}

parece ser persistente y continuo, especialmente bajo los $2500 \mathrm{~m}$ de altura. Los casos que se exponen presentan algunas evidencias arqueológicas determinadas con radiocarbono, dentro del margen de tiempo antes referido. Estas poblaciones debieron enfrentar un ambiente similar al actual, más el aporte de aguas corrientes con caudales más regulares que, en conjunto, ofrecieron un régimen alternado de mayor y menor humedad, permitiendo el desarrollo biótico en cuencas y drenajes que, en última instancia, alcanzaban el Pacífico. Los grupos humanos de esta etapa final del desarrollo precerámico del área debieron acomodarse al paisaje árido en un tiempo postoptimun climaticum, enfatizando el régimen de subsistencia en el cuidadoso control y explotación de pequeños y más amplios nichos ecológicos que se insertan en uno de los espacios más estériles de los Andes. La existencia de estas poblaciones dispersas en los diversos ambientes del área demostraría una densidad suficiente para aceptar que los recursos naturales podían soportar explotaciones en determinadas temporadas.

Se plantea que los diversos microambientes aparentemente no habrían sido capaces de apoyar el desplazamiento de grupos transhumánticos, ofreciendo más bien algunos obstáculos al desarrollo a través de un proceso de aridez que habría inhibido la circulación humana. Sin embargo, se sugiere teóricamente que la población regional, en este rango de tiempo entre los 4000 a 2000 años AC, percibía el espacio con un fino criterio multiecológico. Para este efecto pudieron establecer diversos sistemas de explotación microambiental, que incluyeron el manejo temporal de los recursos establecidos escalonadamente entre la costa y el altiplano andino. La subsistencia regional de los grupos que transitan hacia el comienzo de la formación de los primeros focos horticultores debe entenderse bajo un modo de vida extraordinariamente dinámico, con especial énfasis en esta región, que entre la costa y el altiplano las distancias promedian algo más de $200 \mathrm{~km}$. El conocimiento de la real naturaleza de la dinámica transhumante y su verificación con los contextos estratigráficos de los sitios electos está en proceso de elaboración. 


\section{El ambiente}

Los sitios se ubican en una faja muy restringida entre el altiplano y la costa, manteniendo como eje transversal el paralelo 20, inmediatamente al sur de Pisagua (Figura 1). El área mayor se ha dividido de acuerdo a los actuales ambientes ecológicos, que de uno u otro modo sugieren los caracteres subactuales vinculados con la ocupación prehistórica. Se pueden destacar diversos ambientes que cubren extensiones significativas del área electa:
1) Litoral o Cordillera de la Costa.
2) Cuenca longitudinal.
3) Precordillera.
4) Cuencas interandinas.
5) Altiplano y salares.

Las particularidades físicas de estos ambientes son bien conocidas en la literatura especializada. No obstante, es necesario explicar la disposición de los microambientes que se detectan en la faja descrita en este artículo.

Al colocar los sitios que representan los diversos casos de desplazamientos en un perfil ideal, se aprecia que en un momento más o menos sincrónico hay campamentos establecidos en los ambientes que poseen los recursos naturales de mayor extensión espacial: cuencas interandinas, cuenca longitudinal y litoral. El análisis más detallado señala la existencia de poblaciones muy densas en el microambiente "desembocadura de ríos", en donde se sitúa el primer caso de desplazamiento.

Con cierta proximidad a la costa se sitúa la quebrada de Tiliviche, con recursos de vertientes que alimentan un drenaje superficial y permanente, independiente de la recarga pluvial de la línea divisoria de aguas, emplazada en el alto andino. Esta quebrada con agua potable permanente presenta un conjunto de sitios que caracterizan el segundo caso de desplazamiento.

Las quebradas bajas se han separado del conjunto mayor que se presenta a través de largas quebradas o valles potentes que descienden desde la línea divisoria de aguas hasta el Pacífico. El valle de Camiña, que desemboca en Pisagua Viejo es parte de este conjunto y presupone una comunicación directa entre la costa y el altiplano. Sin embargo, al sur de Camiña se ubican varias quebradas también con ríos alimentados en la alta cordillera, pero sus desagües se disponen en la cuenca longitudinal. La quebrada de Tarapacá, aunque está en una posición de baja altitud, se emplaza inmediatamente sobre la cuenca del Tamarugal. Para los efectos de este artículo se denominan quebradas altas, ya que su continuación hacia el oriente adquiere alzamientos más significativos. El caso 4 de desplazamiento se ubica precisamente en un sector de fricción entre la quebrada de Tarapacá y la cuenca longitudinal inmediata.

Entre el plano inclinado subandino denominado precordillera y el alzamiento de la Cordillera de la Costa se ubica un conjunto de cuencas que adquiere una apariencia de gran valle longitudinal. Varias extensiones de estas depresiones dieron lugar al desarrollo de recursos forestales de extraordinaria importancia para los asentamientos preagrícolas. El tercer caso de desplazamiento se intercala en plena Pampa del Tamarugal, al margen de los microambientes antes referidos.

Finalmente, el caso 5 se sitúa en los microambientes de salares que actualmente se muestran como cuencas cerradas, con cubiertas saladas, dependientes de la particular ecología del altiplano, en donde debieron prevalecer recursos de altura, como la concentración de fauna utilizable. Al apreciar el croquis de microambientes (Figura 1) se verifica que la cubierta estéril predomina el paisaje, y los asentamientos se localizan en donde hay condiciones hídricas suficientes, que pueden coincidir o no con los poblamientos más significativos. En los lugares en donde existió un balance entre los recursos de agua y fuentes de caza-recolección-materias primas se nuclearon temporalmente los sitios que se representan en los casos de este artículo.

Los microambientes actuaron como enclaves ecológicas, en una faja muy sensible a los desplazamientos humanos, estimulados por la proximidad de diversas clases de recursos. Para poder explicar movimientos de flujo y reflujo entre los polos situados en el altiplano y la costa se hace necesario enfatizar mejor las condiciones ecológicas en la zona intermedia. Los recursos de las quebradas cubrieron espacios lineales, normalmente estrechos, a diferencia de las cuencas del Tamarugal que cubrían un hectareaje extraordinariamente mayor. Estos aspectos deben tomarse en cuenta para situar entre ambos polos un sector intermedio con recursos particulares, que facilitaban el desplazamiento en el perfil costa-altiplano. 
Efectivamente, las cuencas cerradas longitudinales reciben el desagüe de varias quebradas locales, con aportes superficiales y subterráneos, reflejando la mayor o menor recarga andina a través de los niveles de los acuíferos subterráneos. En la estación del invierno boliviano (verano) las lluvias aumentan considerablemente, dando lugar a los desagües torrentosos que cubren las pampas intermedias, o que se acumulan bajo la superficie, por la falta de salida al Pacífico. De este modo, en una región absolutamente árida, prácticamente sin registro de lluvias locales, se mantiene una reserva de agua en varios sectores subterráneos del Tamarugal. Las condiciones bióticas de estas depresiones reflejan los caracteres hídricos antes mencionados. Cuando los acuíferos por capilaridad se encontraban cerca de la superficie permitían la extensión de grandes cubiertas forestales de Prosopis, y matorrales, utilizados como forrajes (pillallas y soronas). En tiempos de sequías los acuíferos disminuían su nivel, las cubiertas vegetacionales en especial las plantas de raíces cortas debieron desaparecer, y aun las arboledas de profundas raíces pudieron concentrarse en los niveles más inferiores de las cuencas. En consecuencia, los recursos forestales más que grandes bosques estables deben entenderse como áreas de expansión y contracción reguladas por los desagües andinos.

Estos antecedentes sirven para determinar que en una de las áreas más áridas de los Andes, por lo menos desde fines del Pleistoceno, existieron recursos de agua a través de arroyos exóticos, y desplazamientos subterráneos que activaron irregularmente las cuencas forestales. El aprovechamiento de este ambiente se mide en términos de grandes reservas de recolección de vainas de algarrobos y tamarugos (forraje), y especialización de las prácticas de molienda, con resultados dietéticos básicos (glucosa). Por otra parte, las concentraciones temporales de aguas superficiales permitían depósitos y vegetación lagunar, asociadas a faunas desplazadas a este nivel de base. Tanto las prácticas de caza como de recolección bajo estas condiciones presentan tecnologías simples. Hacia fines del verano y quizás en toda la estación cálida (cosecha), tanto hombres como animales utilizaron las cubiertas forestales, desplazados desde la costa y cuencas interandinas.

El paisaje actual en franco deterioro demuestra que tanto la incorporación de la agricultura en las quebradas como la introducción de tecnologías industriales en tiempos históricos han afectado sensiblemente los microambientes que hemos ubicado al interior de la costa.
Resulta complicado conocer la periodicidad que pudo representar el desarrollo de óptimas condiciones en esta depresión intermedia. Es probable que desde el optimum climaticum hasta ahora hayan existido períodos de sequía que afectaron considerablemente la zona intermedia entre los Andes y la costa. Este efecto debe tenerse en cuenta para sugerir desplazamientos cíclicos en torno a estos recursos, ya que en determinadas épocas la producción de la Pampa del Tamarugal se limitó a escasas cosechas que podrían inhibir o retardar el desplazamiento de grupos. Bajo estas circunstancias, la humedad de la Cordillera de la Costa no fue suficiente para recibir hombres y animales dispuestos a utilizar recursos mediterráneos. Las plantas y animales de la Cordillera de la Costa no alcanzaron un desarrollo considerable, por el déficit de la vegetación de lomas, que en esta zona no ofrece la importancia de la costa peruana. Los escasos oasis de neblinas de la Cordillera de la Costa son muy reducidos en el barranco, al occidente de la depresión intermedia. En consecuencia, cuando los recursos intermedios disminuían o aumentaban, los grupos establecidos entre las tierras altas y bajas detenían o reactivaban sus desplazamientos en el perfil regional.

Es probable que del balance entre microambientes internos con producciones temporales o estacionales y los más permanentes de la costa hayan en conjunto estimulado el flujo de diversos grupos humanos que se especializaron en el establecimiento de circuitos entre las cuencas interandinas, quebradas intermedias, Pampa del Tamarugal y costa. El sitio Conanoxa en la quebrada de Camarones ha demostrado un patrón transhumántico que apoya esta hipótesis de trabajo. Además, en la mayoría de los sitios precerámicos estudiados en la quebrada de Tarapacá se han registrado evidencias de mariscos y pescados trasladados desde el Pacífico. Esta demostración de contacto entre dos pisos lejanos estimula la suposición que similares traslados pudieron efectuarse desde estas quebradas al altiplano inmediato. Las fechas radiocarbónicas de Tarapacá sugieren que existían contactos con la costa desde los 4500 años AC. Las formas en que se llevaron adelante estos contactos y el carácter de los traslados interambientales es, por ahora, un tema en debate.

\section{Los sitios}

En este artículo se considera un conjunto de sitios que representa diversas situaciones en los microambientes del área (Figura 1): 
1) Pichalo y Huelén (costa, con desembocadura de río): 4000 a 2800 años AC.

2) Tiliviche (quebrada baja con vertiente): $4000 \mathrm{a}$ 3000 años AC.

3) Rinconada (Pampa Tamarugal): 3000 a 2000 años AC (?).

4) Tarapacá (pampa-quebrada con río): 4000 a 3000 años AC.

5) Huasco (altiplano): 4000 a 3000 años AC.

Las fechas de Tarapacá, Huelén y Pichalo presuponen un rango de sincronía entre los cinco casos propuestos. Las comparaciones establecidas entre los depósitos estratificados de Tarapacá, Tiliviche y Pichalo demuestran una coherencia concreta. Las fechas de Tarapacá sobrepasan en algo los 4000 años AC (Tarapacá 14 y 2A), y es posible que estas poblaciones hayan persistido en el área hasta fechas cercanas a los 2000 años, registradas en Tarapacá 18. Esta suposición se afirma en la existencia de sitios con dataciones intermedias del orden de los 2800 años AC (Tarapacá 12). Debe establecerse que la ocupación en Pichalo quedaría determinada por los componentes tempranos de la Cultura del Anzuelo de Concha, datado en la costa de Arica hacia los 4000 AC. Los sitios de Rinconada y Huasco no presentan depósitos adecuados para dataciones absolutas, pero a través de sus tipologías podrían vincularse directamente con las industrias de los sitios seleccionados. No es posible aceptar que todos los sitios presenten tiempos comunes, para el planteamiento de la hipótesis central de este artículo. Más bien se trata de sugerir que en un mismo rango de tiempo se establecieron diversos sitios con estadios de desarrollos comunes, que explotaban los microambientes en largos desplazamientos entre tierras bajas y altas.

\section{Pichalo y Huelén}

Punta Pichalo corresponde a un gran campamento costero ubicado en Pisagua, cuyos profundos depósitos de basuras fueron excavados por Bird (1943). A partir de los 4000 años AC se extendió en este sector una población especializada en la explotación del mar, cubriendo importantes sectores de la costa. Estas poblaciones lograron estabilizar su economía a través de la intensificación del aprovechamiento del mar tanto en Pichalo como en los grandes campamentos registrados en Pisagua Viejo (desembocadura del río Camiña). Los recursos marítimos en esta área son cuantiosos y pueden soportar sobrepoblamientos, sin crear desajustes considerables entre ambiente y hombres. Con este punto de vista y salvo una breve temporada del invierno, el ciclo anual pudo concentrarse a través del litoral, incluyendo desplazamientos internos sólo cuando las mareas son alteradas en la estación de invierno. No obstante, en el verano las condiciones para la pesca, caza y recolección son más favorables. Hasta ahora es difícil adjudicar a una estación determinada el tiempo de mayor producción marítima, pero en el litoral de esta región hay cierta tendencia a un aumento considerable de la pesca y caza, cuando en verano los cardúmenes de sardinas se apegan al litoral, atrayendo grandes masas de peces que quedan al servicio del hombre con tecnologías simples. Este fenómeno ocurre en ciertas temporadas del ciclo anual, dejando un tiempo normal de producción menos cuantiosa, que pudo coincidir con el tiempo favorable al desarrollo de la producción mediterránea, estimulando de esta manera el desplazamiento de grupos al interior.

Estos sitios se ubican en la desembocadura de los ríos, por cuanto se trata de sectores que se insertan en la economía marítima, pero a su vez se adaptan a las condiciones favorables creadas por las desembocaduras específicas. Las posibilidades de mantener una población fija son seguras, hasta el punto que pudieron recibir sobrepoblamientos, con periódicos descensos de grupos. En efecto, la producción del mar es independiente de los desajustes ecológicos que puedan haber ocurrido en los microambientes interiores. De modo que sus recursos pueden considerarse dentro de los más cuantiosos del área, y sirvieron en los urgentes procesos de equilibrio dietético, cuando los grupos se vieron afectados por crisis de subsistencia en los nichos interiores.

En la desembocadura del río Loa la situación ecológica en general parece ser similar a Pichalo. Desgraciadamente en Pisagua los patrones de poblamiento se encuentran cubiertos por densas y sucesivas estratificaciones de basuras. A pesar de esto, se puede inferir teóricamente que la relación entre el ambiente marítimo y los hombres había creado una interacción muy estable a través de campamentos convergentes en donde se concentraban diversos grupos desplazados en la costa e interior inmediato. Un tipo de patrón de asentamiento se ha ubicado en la desembocadura del río Loa (Núñez et al. Ms). En este ambiente de desembocadura, una población se 
concentró en los 2800 años AC, aglutinando una aldea con habitaciones de plantas más o menos circulares, hasta cubrir un área de cerca de 100 recintos. Puede asegurarse que este asentamiento representa a una corriente que se extendió en gran parte de la costa, con un equipo muy especializado, derivado de la experiencia de la llamada Cultura del Anzuelo de Concha, inferida estratigráficamente, y del Complejo Chinchorro, reconocido por sus contextos funerarios.

Huelén presenta, al igual que algún momento temprano de Pichalo, un proceso migratorio que se concentra en la costa con eficiencia de desembocadura y una tecnología especializada en la explotación pesquera, que más tarde pone énfasis en la recolección de mariscos. La incorporación de estos grupos a la costa y el nucleamiento gradual de la aldea presupone un incremento progresivo de población, tanto así que evidencias notables de este patrón se han detectado en Taltal (Mostny 1964), dejando estaciones semialdeanas intermedias en diversos sectores de la costa sin desembocaduras. Huelén representaría un caso concreto de desplazamiento humano que se localiza en sectores eficientes de la costa, sin evidencias hasta ahora conocidas de aprovechamientos de microambientes al interior. La falta de algarrobo, tamarugo y de otros productos internos sugieren que el equilibrio alimenticio con la costa era más que suficiente. La presencia de maderas duras de Prosopis y de muy escasas semillas de algarrobo en algunas estructuras pudieran reflejar cierta explotación de la vegetación ubicada en la propia desembocadura del río Loa. Los exámenes estratigráficos de Huelén no demuestran rasgos culturales vinculados con traslados de grupos hacia el interior de la costa. Más que una dinámica vertical, el caso Huelén sugiere intensos movimientos horizontales a través del litoral, manteniendo como eje a los distintos focos aldeanos que se establecieron en enclaves costeros. La población Huelén (portadora de las finas hojas Taltal) depositaron las basuras iniciales hacia los 2800 años AC. Asociadas a esta muestra se encontraron algunos restos culturales procedentes de las tierras altas: fragmentos de obsidiana, y pequeñas plumas verdes que pueden corresponder a "pericos cordilleranos", comunes hoy en día en el altiplano del área que se estudia. Es difícil asegurar que estas evidencias sólo aparecen en las primeras basuras, pero los registros constatan esta situación sólo en la primera ocupación. Se sugiere que grupos portadores de este patrón habitacional se incorporaron a ciertos enclaves costeros, constituyendo parte de un proceso migratorio entre tierras altas y bajas. De uno u otro modo, el caso Huelén ejemplifica una adaptación especializada en producción costera, con una mínima dependencia con respecto a los microambientes interiores. Por cierto que no se propone un carácter de sedentarismo riguroso, ya que el ámbito Huelén incluyó microambientes aledaños como la Cordillera de la Costa en donde cazaron guanacos y ciervos (Hipocamelus antisiensis). La falta de sitios con este patrón en los microambientes interiores prospectados, en los marcos de esta hipótesis de trabajo, sugiere que el entendimiento de los grupos Huelén con la costa excluía la posibilidad de traslados por razones de subsistencia hacia ambientes foráneos. En el río Loa entre los 2000 a 3000 m.snm se han registrado sitios con viviendas tempranas, pero su exacta relación con Huelén no ha sido confirmada debidamente.

\section{Tiliviche}

En la quebrada de Tiliviche hemos registrado varios sitios precerámicos entre los que se destaca Tiliviche 1-b que representa el segundo caso de desplazamiento. Se trata de un gran campamento emplazado a base de viviendas semisubterráneas preparadas con huecos de depósitos y postes. Un cálculo aproximado establece que algo más de 100 depresiones habitacionales se prepararon en la base estéril de la terraza, dejando montículos y cubiertas de basuras con restos de mariscos y abundantes artefactos líticos en superficie. La estratigrafía clarifica que importantes rubros de alimentos marítimos eran transportados desde el litoral inmediato (Pisagua), directamente al sitio, distante más o menos a 40 $\mathrm{km}$. Por otro lado, se registran abundantes restos de fibra vegetal (totora), y una cifra extremadamente alta de manos y morteros. Esto significa que los recursos locales eran utilizados por la población del campamento. La quebrada de Tiliviche se caracteriza por constituir un nicho ecológico restringido, pero valorado por la presencia de vertientes con agua potable que se escurre en un tramo cubierto por densa vegetación utilizada por hombres y animales. Junto a los frutos de Prosopis se debe señalar la abundante presencia de totora en los depósitos arqueológicos y que actualmente crece en las zonas más húmedas del fondo de la quebrada. Las raíces de totora constituyen un excelente alimento que debió extraerse cuando se apropiaban de fibras adecuadas (materia prima) para múltiples manufacturas. Las manos y morteros presuponen viejos hábitos alimenticios similares a las evidencias de prácticas de molien- 
das de Tambillo y Puripica en la zona de Atacama. En este microambiente de Tiliviche con suelos y humedad adecuados para la horticultura es factible la experimentación de plantas trasladadas dentro del esquema de desplazamientos transhumánticos. El control estratigráfico de Tiliviche (Núñez et al. Ms) determina claramente la posición temprana de maíces, que actualmente se analizan por dos especialistas. Es posible asegurar que la población de Tiliviche, que dio lugar a los estratos medios, utilizó el maíz con un carácter experimental, dentro de un contexto preagrícola.

En competencia con la recolección mayoritaria y los primeros intentos de adaptación del maíz temprano se ha logrado un completo inventario de puntas estrechamente vinculadas con prácticas de caza local. Los restos de pieles de camélidos, aves y roedores enseñarían el aprovechamiento de animales atraídos por las vertientes, pero los análisis cuantitativos de basuras descartan este rubro como indispensable o de presencia significativa. Esta misma metodología establece que los rubros de alimentos marítimos trasladados al nicho son superiores y complementan a los recursos locales. La densidad de los campamentos ajustados a este mecanismo de ensamble de recursos es evidente desde los sitios de Saya, Quiuña Bajo, Quiuña, Aragón y Tiliviche registrados por el autor.

Si este nicho no ofrecía cuantiosos recursos alimenticios, hay que deducir de sus depósitos estratigráficos las posibles causales de por lo menos cinco campamentos en el sector de Tiliviche. Las razones parecen ser complejas: subsistencia temporal, uso de materias primas locales y estacionamientos previos al ingreso de la costa, pampas y quebradas altas.

Las plantas silvestres, incluyendo los frutos de Prosopis, sirvieron como fuentes de alimentación, pero la abundancia de fibras de totora en los depósitos indicaría una recolección destinada a tratamientos artesanales. La aplicación en cuerdas, vestimentas, bolsos, esteras, usos habitacionales, funerarios, etc., está bien documentada en la costa e interior. Pero los totorales de la desembocadura del río Camiña, obviamente que habrían sido suficientes para las exigencias locales. Hay otros recursos internos de mayor atracción como fueron los forestales y materia lítica adecuada para la elaboración de equipos. En este sentido hay un especial cuidado en seleccionar litos para transformarlas en artefactos de uso local y foráneo. Está claro que partes importantes del campamento fueron talleres líticos, proveedores de industrias trasladadas con los grupos en el perfil costa-altiplano. Esta hipótesis tiene validez con algunos instrumentos procedentes de la quebrada de Tiliviche que, sin duda alguna, fueron usados en la costa de Pisagua Viejo. La posibilidad de que existieran nichos con un mínimo nivel de alimentación local, asociado a potentes reservas de litos, ofrecería la posibilidad de incrementar los medios de producción. Es difícil saber por ahora qué artefactos se usaron exclusivamente en los campamentos de Tiliviche, pero la estratigrafía habla a favor de un énfasis local en el manejo de recursos vinculados con la preparación de fibras (cuchillos), caza (puntas), preparación de maderas (cepillos), moliendas (manos y morteros), etc. Sin embargo, es un hecho tipológicamente cierto que existieron herramientas comunes a diversos nichos (v.gr., puntas de bases escotadas), del perfil regional. El registro de una barba lítica de arpón compuesto entre la industria del campamento Aragón en plena Pampa del Tamarugal, y un anzuelo lítico también en proceso de elaboración bajo el patrón del modelo de concha (Tiliviche I-b), inducen a aceptar que en estos campamento se preparaban instrumentos de uso foráneo.

Los inventarios estratigráficos de Tiliviche han asegurado la presencia del anzuelo de concha, a consecuencia del desplazamiento de grupos que en la costa específica conformaron la "Cultura del Anzuelo de Concha" (Bird 1943), en su fase tardía. Esta situación ofrece una nueva inferencia ecológica. Esto es, que junto a los grandes campamentos marítimos portadores de anzuelos de concha (campamentos convergentes de Pisagua Viejo y Pichalo) se establecían campamentos divergentes en diversos nichos interiores, bien representados con el caso 2 de Tiliviche.

El ascenso de grupos a las quebradas y Pampa del Tamarugal tiende a demostrarse con evidencias estratificadas en varios campamentos en estudio. Por otra parte, las excavaciones en Tarapacá (True et al. 1970) ya habían recomendado que la prolongación final del desplazamiento sobre el nivel de base regional (cuenca longitudinal) pudieron alcanzar la costa inmediata. Se supone que el Tamarugal no fue un centro receptivo exclusivo, sino más bien se considera como un área de recursos intermedios entre el potencial andino y marítimo. Con este antecedente ecológico, no es muy irreal sugerir que los hombres dejaron herramientas comunes en los 
diversos microambientes, y otras diferenciadas de acuerdo a los procesos de especialización que implica la explotación en ecologías contrastadas.

Es difícil señalar una estación concreta para el ascenso hacia los campamentos del caso 2. Quizás si en algún momento del verano o invierno, cuando maduran los frutos de Prosopis, aconteció el tráfico con cierta regularidad. Pero no debe descartarse el antecedente que dice relación con la irregularidad temporal de las condiciones favorables en el Tamarugal. No todos los años fueron tiempos de buenas cosechas, ni tampoco existió una permanente y lozana vegetación con fauna utilizable. Lo importante en este caso es que los grupos pueden ocupar la costa con tecnologías especializadas, y a su vez perciben el uso de los microambientes interiores como partes de un solo universo, cuando existen condiciones favorables. En la medida que estos circuitos no ascendían de la cuenca longitudinal, la alimentación de origen marítimo podía servir de apoyo indispensable. Al oriente de la cuenca disminuyen considerablemente los restos de pescados y mariscos, en tanto que la fauna y recolección local adquieren mayor significado.

No es posible pensar que tan complejo sistema de utilización de nichos haya ocurrido sólo al interior de Pisagua. Es casi seguro que este esquema transhumántico tiene duplicados en Camarones (v. gr, Conanoxa), con dataciones bien determinadas por Niemeyer y Schiappacasse (1963) que no pasan de los 2000 años AC. Entre los 4000 a 2000 años AC pudo desarrollarse un flujo poblacional entre tierras altas y bajas con desplazamientos recíprocos entre quebradas bajas y cuenca longitudinal con la costa aledaña. El modelo parece presentarse en su fase temprana a través del caso 2 (Tiliviche), y una situación más tardía o final se ubicaría en Conanoxa.

\section{Rinconada}

Este sitio podría representar a varias evidencias dispersas en Pampa del Tamarugal. Se trata de un sector poco concentrado en el borde occidental de la cuenca longitudinal, al oeste de Tirana (Núñez Ms.). La industria lítica se ubica sobre la costra actual, entre arboledas relictuales, en un ambiente típico del Tamarugal, fuera del ámbito de las quebradas. Los artefactos con formas definidas son escasos, pero tanto las puntas, cuchillos y cepillos grandes indican una explotación local de Prosopis.
El caso 3 se individualiza por el carácter más temporal que adquieren los asentamientos asociados directamente a los recursos forestales. Puede sugerirse que son más bien paraderos reocupados constantemente, localizados por la presencia de áreas boscosas sin apoyo directo de vertientes. A estas localidades alcanzaron grupos costeros, de quebradas y tierras altas, en reducidos lapsos a juzgar por la falta de estructuras y acumulaciones de basuras. Aunque no está bien cuantificado, la mayor cantidad de vainas de algarrobos y tamarugos se desprenden en verano, en coincidencia con la estación de lluvias y mal tiempo cordillerano que afecta desde los 2500 a 4000 m.snm.

\section{Tarapacá}

En el tramo inferior de la quebrada de Tarapacá, sobre cotas superiores a la cuenca longitudinal, se sitúan varios campamentos con dataciones fluctuantes entre 4500 a 2000 años AC. Las causas de estos asentamientos guardan relación directa con el usufructo de un microambiente constituido por el desarrollo de vegetación y fauna a lo largo del tramo final del arroyo Tarapacá y su fricción con arbustos $\mathrm{y}$ arboledas de la cuenca del Tamarugal. Es probable que en el fondo de la quebrada emergiera alguna vertiente tipo Huarasiña, pero la mayor humedad del área es el resultado de los escurrimientos superficiales y subterráneos procedentes de la línea divisoria de aguas. De modo que el drenaje estaba regulado por la mayor o menor recarga pluvial cordillerana, estimulándose la movilidad de grupos en la medida que los recursos hídricos disminuían temporalmente. Inversamente, las grandes inundaciones de verano lograron atraer contingentes a Pampa del Tamarugal. Por esto, el caso 4 integra tanto a la cuenca longitudinal como a las quebradas bajas en un solo universo de explotación. La movilidad de grupos surge aquí por el interés en controlar las cuencas forestales y quebradas aledañas, a partir de campamentos más o menos estables emplazados exclusivamente en el sector en que la quebrada de Tarapacá se diluye en la cuenca longitudinal. Estos campamentos abiertos se han fechado con restos orgánicos de depósitos estratificados de poca profundidad que en ningún caso se alteraron por humedad de origen pluvial. Prácticamente todas las evidencias arqueológicas aseguran que la cubierta de los campamentos fechados desde los 4500 años AC no recibieron lluvias significativas, y que el régimen de vigencia desértica estaba presente, con un drenaje escurrido desde los Andes a través de la quebrada de mayor potencia 
al tiempo actual, pero con gastos irregulares. La especialización del crecimiento de las arboledas autóctonas presupone una eficaz ambientación a un paisaje desértico alternado de grandes aportes hídricos temporales. En efecto, los tamarugos tienden a crecer o germinar en condiciones de máxima humedad subterránea y superficial, hasta consolidarse con las raíces incipientes. El desmesurado desarrollo de la raíz permite capturar los acuíferos subterráneos, en etapas en que el nivel de humedad era más bajo por una menor recarga cordillerana (sequía). Por otra parte, el follaje actúa como malla condensadora de neblinas rasantes recargadas con humedad desde el Océano Pacífico, de modo que un particular proceso de asimilación nocturna y respiración diurna, sin regadíos convencionales, sirvió para mantener densas arboledas y hombres, insertos en uno de los desiertos más secos utilizados por grupos transhumantes.

Tarapacá (caso 4) representa bien a las concentraciones de campamentos en los sectores bajos de las quebradas junto a la cuenca longitudinal, en donde se agrupaban cuando las cosechas del Tamarugal y en los propios cursos inferiores eran altamente productivas. Si hasta aquí alcanzaron los grupos del caso Tiliviche es cuestión discutible a pesar de que las habitaciones semisubterráneas con postes, abiertas en la base estéril de las terrazas, las basuras con restos marítimos y los conjuntos de herramientas diagnósticas, son comparables con el caso presente. El acceso de gentes llegadas desde las cuencas interandinas (v. gr., Huasco) a constituir partes del caso Tarapacá, también se deduce de inferencias tipológicas y etnológicas. Hasta no hace un tiempo, los pastores de la laguna de Huasco preferían organizar sus descensos al oasis de Pica (distante a dos jornadas diarias a pie) en la temporada de verano. Se pueden agregar algunas observaciones más intituivas que ornitológicas, en el sentido de que las aves bajan desde las tierras altas a nichos bajos, cuando el "invierno boliviano" altera el ambiente andino.

Los campamentos que componen el caso Tarapacá se caracterizan por débiles depósitos, con escasos restos de mariscos y pescados, pero son más frecuentes los registros de vegetales locales y fauna mayor (guanacos). No obstante, no debe excluirse la presencia de animales más pequeños, similares a los registros de Conanoxa. Hay sin duda alguna un equilibrado entendimiento con los recursos de la quebrada, la cual presenta un hectareaje mucho más extenso al caso Tiliviche. La presencia de manos y morteros afirma lo anterior, en un contexto de herramientas de caza y recolección (True et al. 1970). Los campamentos pudieron controlar grandes extensiones de la cuenca y quebrada, para lo cual debieron establecerse en un lapso mayor a través de asentamientos más estables con un típico patrón habitacional en leves depresiones, nucleadas en pequeños racimos (True y Núñez Ms.). Los escurrimientos superficiales en la quebrada de Tarapacá estimularon el desarrollo de ciertos cultivos, transportados por grupos transhumantes (caso 4); el registro de maíz en un sitio eventualmente temprano en este sector de la quebrada (True et al. 1970) debe ahora valorizarse a la luz de las evidencias estratigráficas tempranas del sitio Tiliviche I-b.

\section{Huasco}

Varios campamentos abiertos se han ubicado en los bordes de la cuenca de Huasco, vinculados con recursos interandinos diferentes a los casos antes referidos (Núñez y Varela 1966). El caso 5 presupone el desarrollo de asentamientos transitorios en cuencas y altiplano inmediato, en la zona de división de aguas, sobre techos altos de 3500 m.snm. La cuenca de Huasco presenta en la actualidad varias vertientes y desagües de verano que se encierran en la depresión, hasta configurar un nivel lacustre, como pequeña laguna con vegetación y fauna abundantes. Sin duda, la naturaleza lacustre debió ser mayor a la actual si el régimen de precipitaciones andinas fue superior en etapas subactuales. Recientemente, todo este sector se consideraba entre los más importantes centros de caza, y al igual que otros salares altos, un elenco de numerosas puntas se encuentran en las playas subactuales. Se trata de un ambiente adecuado para las prácticas de caza más que cualquiera otra forma de apropiación. Los suelos actuales se destinan a mantención de ganado camélido (forraje duro), sin cultivos de altura. Es muy difícil aceptar que algunas tuberosas y gramíneas crecieron con la misma extensión y calidad similar a las cuencas entre Cariquima e Isluga, pero si algún remanente fue recolectado, esto debió ocurrir después de la estación de verano. El efecto del "invierno boliviano" (verano) favorece los asentamientos a lo largo de cierto tiempo del ciclo anual, este factor restringe la ocupación a estaciones favorables. Por esto, los campamentos están bien determinados por la concentración lítica, sin huellas superficiales de depósitos ni restos de estructuras habitacionales (?). 
El microambiente de Huasco probablemene se habría utilizado como área de caza y pastoreo, adecuado para la apropiación de cueros, carne, huesos, lana, huevos, plumas, etc., en alguna temporada favorable. La presunción que ocuparon otros microambientes del altiplano, quebradas y costa, se debería a que la producción especializada de las cuencas interandinas resultaría insuficiente, desequilibrada y estacional, para mantener a densos contingentes humanos con estabilidad en campamentos fijos (alteración en verano).

La falta de prospecciones con objetivos específicos no ha logrado cubrir el déficit de información sobre sitios precerámicos con manos y morteros en tierras altas. Los registros establecidos en las cercanías de los oasis de San Pedro de Atacama (Le Paige 1970) no pueden ser los únicos, sino más bien cubrirían importantes áreas del Loa superior y cabeceras de los valles altos al norte de la Puna de Atacama. Tiliviche 1-b demuestra que estas ocupaciones se extendieron por amplias regiones, como partes de un estadio de desarrollo panandino. Algunos sitios de esta naturaleza como Puripica y Tambillo parecen haber formado parte de circuitos transhumánticos que se regionalizaron en los diversos pisos ecológicos de la Puna de Atacama, con débiles contactos con la costa por la clara ausencia de valles entre la puna y el Pacífico. Sin embargo, el movimiento de grupos aquí actuó entre las extensas áreas de depresiones altas (lagos, mesetas y cabeceras de ríos) fluctuantes entre los 3500 a $4500 \mathrm{~m} . \mathrm{snm}$, en la zona de los nevados y conos volcánicos y la cuenca específica de Atacama, que conformó el nivel de base regional atrapando el drenaje en grandes depósitos lacustres alternados por áreas saladas. Entre ambas zonas potenciales en términos de forraje y recursos en general se agregan en los bordes de la cuenca baja de Atacama (2400 m.snm) diversos oasis con arboledas de Prosopis, que en principio recuerdan similares respuestas a los recursos de la Pampa del Tamarugal. Entre ambos pisos poco distantes pero contrastadamente diferentes en altitud se ubican las quebradas con recursos y pasos intermediarios. Las ocupaciones humanas pudieron establecerse en las depresiones altas en las estaciones o ciertas temporadas fluctuantes entre invierno y verano. Por lo menos es casi seguro afirmar que los asentamientos no pudieron establecerse en la estación de verano o invierno por el mal tiempo cordillerano. Areas destinadas preferentemente a trabajos de caza eran abandonadas por el aprovechamiento de microclimas más cálidos en quebradas bajas, oasis y playas deprimidas en el actual Salar de Atacama (v. gr., Tambillo). Aquí variaban los recursos con un excelente balance entre actividades de caza y recolección vegetal. Hasta ahora tanto hombres como aves y animales descienden a pisos más bajos, al comienzo del llamado "invierno boliviano" (verano). Los grupos transhumánticos establecieron circuitos para el aprovechamiento de los tres ambientes básicos: cuencas altas, quebradas intermedias y cuencas bajas (Salar de Atacama), distribuyendo tiempo y energía en diferentes nichos, dentro del ciclo anual. La falta de drenaje al Pacífico aquí oculta niveles de relaciones específicas con los casos de Tarapacá, pero el uso estacional o temporal de los diversos pisos de la puna en términos de alimentación y forraje es parte de un desarrollo común (proliferación multiecológica de manos y morteros).

\section{Las industrias líticas}

Una amplia comparación de las industrias líticas representadas en los sitios del perfil costa-altiplano, a través de los casos seleccionados en esta discusión, indica un parentesco global. Por ejemplo, hay puntas y cuchillos similares en Pichalo (Bird 1943) y Tiliviche (Núñez et al. 1972-3). Las puntas y cepillos de Rinconada se asemejan a Tiliviche. Las puntas lanceoladas, triangulares, pedunculadas y de bases escotadas de Huasco (Núñez y Varela 1966), duplican iguales formas de Tiliviche.

Los campamentos del caso Tarapacá tienen herramientas diagnósticas que se han reconocido entre el altiplano y la costa. Algunas dataciones sirven para situar mejor la cuestión cronológica. Tarapacá-12 se ha fechado hacia los 2800 años AC con puntas lanceoladas, hojas-cuchillos, puntas de bases escotadas y manos de molienda. En Tarapacá 2-A hay un elenco de puntas bien presionadas de formas lanceoladas, con pedúnculos redondeados y bordes finamente presionados fechados en 4000 años AC. Especímenes similares se registran en varios campamentos que componen el caso Tiliviche. Hay varias razones tipológicas que afirman la ocurrencia de artefactos comunes en los diversos casos presentados. Pero a su vez, este esquema de trabajo sugiere que en cada nicho específico se utilizaron herramientas especializadas y diferenciadas de los patrones comunes. El examen taxonómico tradicional podría inferir 
diversas fases culturales, o desarrollos tecnológicos independientes, donde solamente se trata de componentes líticos especializados en los diferentes microambientes del perfil. La identificación de las formas diagnósticas y sus relaciones con las herramientas comunes o multiecológicas es complicada, en la medida que la falta de dataciones radiocarbónicas no avala una seriación válida para todo el perfil. Al margen de esta problemática se plantean algunos ejemplos de formas especializadas en determinados microambientes:

Huelén: Presencia de largas puntas con bases levemente estrechas o pedúnculos apenas esbozados, utilizados en la caza de arponeo con cabeceras desconectables. Grandes hojas-cuchillos taltaloides usadas al parecer en la preparación de grandes animales marinos.

Pichalo: Enfasis en la elaboración de puntas con aletas sobresalientes curvadas en dirección al fino pedúnculo central, utilizadas en el arponeo de peces menores que requieren una mayor fijación con aletas agudas que actúan como barbas que aseguran la presa móvil.

Tiliviche: Aumento de puntas de formas lanceoladas, cepillos, manos y morteros, vinculados con recursos locales que ya antes se han discutido.

Huasco: Predominio de puntas de base escotadas y lanceoladas, pequeños raspadores y microlitos aptos para raer y cortar (especialización en fauna de laguna).

Tarapacá: En este caso se destacan frecuencias discrepantes con los casos anteriores. Hay un claro predominio de puntas lanceoladas sobre formas pedunculadas, y si esta diferencia se deriva de algún rango de especialización se excluiría el factor cronológico como único elemento de análisis.

El análisis comparativo de las formas comunes entre los casos presentados se basa en la tipología de Tiliviche. Efectivamente, aquí se ha agrupado un total de 44 tipos separados en las siguientes categorías funcionales: puntas (10 tipos), perforadores (1 tipo), cuchillos (9 tipos), raspadores (11 tipos), raederas (1 tipo), cepillos (10 tipos), manos (1 tipo), morteros (3 tipos). De este conjunto se han separado 15 tipos diagnósticos por popularidad y se comparan con los sitios restantes (Moragas 1973).

\begin{tabular}{|c|c|c|c|c|}
\hline Tipos & Pichalo & Tiliviche & Tarapacá & Huasco \\
\hline Raspador plan convexo & $\mathrm{X}$ & $\mathrm{X}$ & $\mathrm{X}$ & $\mathrm{X}$ \\
\hline Punta triangular escotada & $\mathrm{X}$ & $\mathrm{X}$ & $\mathrm{X}$ & $\mathrm{X}$ \\
\hline Punta lanceolada media & $\mathrm{X}$ & $\mathrm{X}$ & $\mathrm{X}$ & $\mathrm{X}$ \\
\hline Doble punta & $\mathrm{X}$ & $\mathrm{X}$ & & $\mathrm{X}$ \\
\hline Punta pedunculada & $\mathrm{X}$ & $\mathrm{X}$ & $\mathrm{X}$ & \\
\hline Punta larga escotada. & & $\mathrm{X}$ & $\mathrm{X}$ & $\mathrm{X}$ \\
\hline Punta lanceolada pequeña & & $\mathrm{X}$ & $\mathrm{X}$ & $X$ \\
\hline Punta lanceolada grande & & $\mathrm{X}$ & $\mathrm{X}$ & $\mathrm{X}$ \\
\hline Cuchillos medialunados & & $\mathrm{X}$ & $\mathrm{X}$ & $X$ \\
\hline Cuchillos elípticos & & $\mathrm{X}$ & $\mathrm{X}$ & \\
\hline Cuchillos convergentes & & $\mathrm{X}$ & $\mathrm{X}$ & \\
\hline Morteros & & $\mathrm{X}$ & $\mathrm{X}$ & \\
\hline Manos & & $\mathrm{X}$ & $\mathrm{X}$ & \\
\hline Cepillos & & $\mathrm{X}$ & $\mathrm{X}$ & \\
\hline $\begin{array}{l}\text { Puntas pedunculadas } \\
\text { asimétricas }\end{array}$ & & $\mathrm{X}$ & & \\
\hline
\end{tabular}

Aunque esta muestra comparativa puede cuestionarse por no considerar la totalidad de los tipos descritos para los diversos casos, la limitación debió ajustarse a las breves descripciones líticas de Pichalo, pero si se acepta como una muestra de análisis, las relaciones tipológicas entre los microambientes son sugerentes:

\begin{tabular}{lr}
\hline Costa-quebrada baja & 7 rasgos comunes \\
Costa-quebrada alta & 4 rasgos comunes \\
Costa-altiplano, & 4 rasgos comunes \\
Quebrada baja-alta & 13 rasgos comunes \\
Quebrada baja-altiplano & 8 rasgos comunes \\
Quebrada alta-altiplano & 7 rasgos comunes \\
\hline
\end{tabular}

A grandes rasgos se aprecia una tendencia "común" entre las industrias establecidas en las quebradas que contactan con la cuenca longitudinal. De esta manera Tiliviche podría formar parte del mismo patrón transhumántico que los sitios fechados en Tarapacá (True y Núñez Ms). Las relaciones entre estos dos sitios y el altiplano (Huasco) indican frecuencias altas que deben tomarse en cuenta en la discusión sobre la real amplitud de los circuitos. Sin embargo, las vinculaciones entre la costa y las tierras altas, sean quebradas o altiplano, son sensiblemente bajas. Sin duda que esta discrepancia puede explicarse de varias maneras si se desea salvaguardar el marco teórico. La economía mediterránea implicaría el uso de conjuntos líticos más o menos similares entre quebradas y altiplano, e inversamente en la costa las herramientas serían más especializadas a consecuencia de las actividades de pesca-caza-recolección diferentes al interior inmediato. 


\section{Hipótesis de transhumancia}

Los trabajos de Lynch (1967) en los Andes Centrales aportaron un nuevo nivel teórico e interpretativo del proceso de adaptación en las tierras altas. Para este efecto estudió las industrias líticas ubicadas entre la alta cordillera y la costa, en pasadizos intermedios, como un medio de "interceptar" posibles circuitos transhumánticos entre tierras altas y bajas. Parece haber consenso que los grupos del Precerámico Tardío percibían el espacio andino con un criterio dinámico, utilizando diversos pisos con sistemas de explotación multiecológica. El manejo alternado de nichos permitió el desarrollo de campamentos transitorios dispuestos en el perfil costa-altiplano, con diferentes categorías de permanencia. Sin embargo, no se sabe mucho de la real naturaleza estacional y/o periódica de los desplazamientos en relación a los diversos casos de circuitos enunciados pero no comprobados.

Lynch (1967) advierte, con razón, que las relaciones entre la costa y las tierras altas deben abarcar algunas formas de desplazamientos transhumánticos, verificables a través del examen de áreas intermedias. Un esquema algo similar se aplicó en la quebrada de Tarapacá (True et al. 1970), aunque el análisis surgió independientemente del criterio de Lynch (1967), válido en los Andes Centrales. Efectivamente, en Tarapacá se trataba de establecer una secuencia y lateralmente tanto el examen tipológico como ecológico recomendó la observación de posibles desplazamientos hacia la cuenca del Tamarugal y costa inmediata. Una de las consecuencias del Proyecto Tarapacá fue la prospección dirigida que uno de los autores efectuó entre Pampa del Tamarugal y la costa inmediata, al occidente de la quebrada homónima (Núñez et al. 1972-3). La elección de la zona intermedia entre Quiuña a Tiliviche y Aragón se definió como un medio de interceptar posibles circuitos entre tierras altas y bajas. Las evidencias presentadas (cinco casos) son parte de esta investigación en desarrollo. Queda fuera de duda que las relaciones entre Pichalo-Tiliviche-Tarapacá son seguras, en tanto que Huasco aún no fechado podría incluirse muy tentativamente. Huelén es un caso de patrón migratorio, al margen de un posible análisis transhumántico.

Al evaluar algo más de 15 sitios reunidos en cinco casos, se sugiere el siguiente instrumento de trabajo:

Caso 1. Grupos se establecen en zonas de desembocaduras junto al Pacífico y no requieren de desplazamientos interiores (Huelén). ubicación

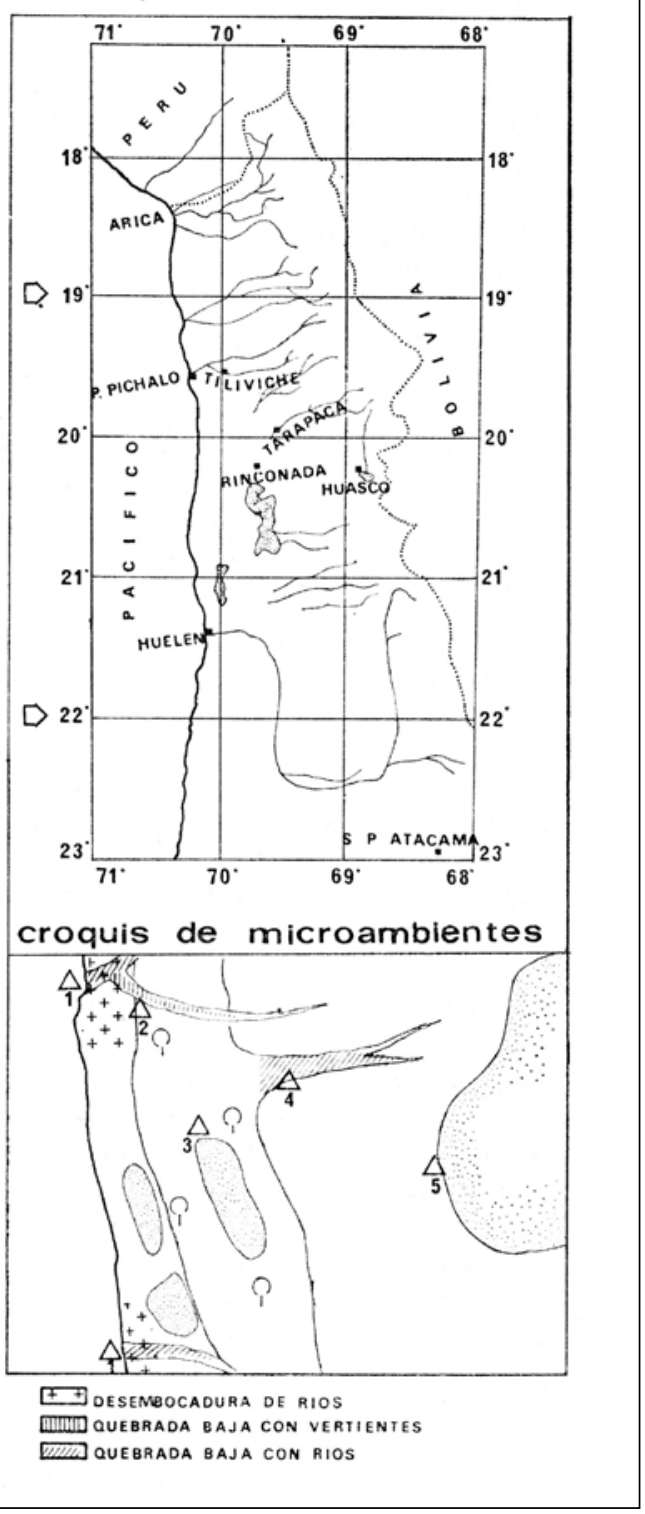

Figura 1. Ubicación y croquis de medio ambientes.

Grupos de Pichalo y Pisagua Viejo emplazan campamentos convergentes en las desembocaduras y ascienden a nichos interiores en temporadas que pudieron regularse por las mareas adversas de invierno. La información etnológica señala que en la costa de Pisagua los mariscadores de comienzos de siglo dejaban el litoral en "las mareas malas" de invierno, y subían a los nichos de Saya y Quiuña (vertientes) en donde recolectaban fibras de totora y capturaban camarones. La prolongación hacia nichos cercanos a la Pampa del Tamarugal constituye el caso siguiente. 
Caso 2. Grupos de la costa abandonan el litoral por una temporada mayor al caso anterior y se instalan en los nichos ubicados en quebradas aledañas a la Pampa del Tamarugal, incluyendo distintas porciones de las cuencas forestales. La subsistencia es suplementada con alimentos trasladados del litoral en campamentos más estables vinculados con recursos locales de diversa índole. Las inundaciones de verano habrían activado el poblamiento entre el fin del verano a fines del invierno (mareas adversas). Tentativamente sería éste el lapso más apropiado para la ocupación de dos principales campamentos con manos y morteros de Tiliviche y Tarapacá.

Caso 3. Grupos de la costa y tierras altas en general acuden bajo un patrón disperso a múltiples porciones de las cuencas forestales por intereses alimenticios en el tiempo de la mayor cosecha de fines de verano y se proveen de maderas duras (Prosopis). Aunque es difícil conocer sus procedencias (prolongación del caso anterior), se advierte aquí una clara separación de la explotación en quebradas. Estos campamentos transitorios tienden a colocarse en el borde occidental de la Pampa del Tamarugal (v. gr., Rinconada).

Caso 4. Grupos que componen el caso 2 ocupan quebradas y cuencas forestales desde campamentos semiestables reocupados periódicamente (v. gr., Tiliviche-Tarapacá) y ascienden a pisos altos entre las quebradas de Tarapacá y Camiña, hasta alcanzar nichos altiplánicos (Cariquima-Isluga) y cuencas interandinas (Huasco). Este desplazamiento coincidiría entre primavera y otoño, tiempo en que la producción del tamarugal baja considerablemente, el drenaje se normaliza, y en la costa hay menos actividad comparativamente con el verano. El caso 4 demostraría el ascenso de grupos Tiliviche y Tarapacá, que dejarían componentes en Huasco. Una alternativa quedaría expuesta si se acepta que los grupos Huasco bajan a otros nichos, reflejando una presión demográfica que actuaría desde tierras altas a bajas, pero esta cuestión es demasiado complicada como para tratarla aquí (descenso en verano).
Seguramente que las inferencias estacionales pueden cuestionarse. No obstante, el hecho de que los desagües estén regulados por una estación lluviosa de verano, en un perfil con contrastes altitudinales, pudo estimular el desplazamiento de grupos en cualquiera de las categorías o casos expuestos. Más que identificar una regularidad estacional, o delimitar circuitos transhumánticos rígidos entre altiplano y costa, debe esperarse más bien la ubicación de sitios con industrias y depósitos comparables, sujetos a datación absoluta. El análisis de universos comparables entre diversos nichos ofrecerá una buena ventaja metodológica. El patrón de desarrollo transhumántico está demostrado claramente con el caso 2 , pero lo que está en discusión son las variables o categorías de transhumancia entre tierras altas y bajas (casos propuestos).

Lynch (1967 Ms) ha expuesto que uno de los problemas vigentes más atractivos sobre transhumancia cultural se relaciona con el registro de evidencias de plantas cultivadas, en los marcos de la emergencia de los primeros focos horticultores. Por cierto que el traslado interambiental pudo mover tanto a hombres como animales y plantas. Un mínimo cálculo botánico asegura que los nichos con recursos de aguas en quebradas bajas sirvieron para adaptar a cultivos del complejo tropical-semitropical (caso 2). La posición estratigráfica de los maíces tempranos de Tiliviche, asociados a una industria de caza-recolección, confirman las sugerencias de Lynch. La presencia de otra evidencia de maíz temprano(?) en Tarapacá (True et al. 1970) adquiere ahora una revaloración significativa.

En la actualidad algunos sitios considerados como "casos" se encuentran excavados y su información está en proceso de redacción. Este artículo refleja la situación especulativa derivada del marco teórico, aún falta documentar varios sitios que seguramente rectificarán algunas apreciaciones. La investigación continúa en estos términos. 


\section{REFERENCIAS CITADAS}

BIRD, J., 1943. Excavations in Northern Chile. Anthropological papers of the American Museum of Natural History, XXXVIII, part IV, pp. 171-316. Nueva York.

- 1967. Muestras de radiocarbono de un basural precerámico de Queani, Arica. Boletín Sociedad Arqueológica de Santiago 4: 13-16.

LYNCH, T., 1967. The nature of the Central Andean Preceramic. Occasional Papers of the Museum State and University, 21, Pocatello.

1967 Ms. Algunos problemas básicos del estadio de cazarecolección andina: Transhumancia. Congreso del Hombre Andino, Arica, Iquique, Antofagasta.

LE PAIGE, G., 1970. Industria lítica de San Pedro de Atacama. Coedición Orbe y Universidad del Norte, Santiago.

MORAGAS, C., 1973. Taxonomía de la industria lítica del sitio Tiliviche 1-b. Memoria de Título Departamento de Historia, Universidad del Norte, Antofagasta.

MOSTNY, G., 1964. Epistolario de Augusto Capdeville con Max Uhle y otros arqueólogos e historiadores. Arqueología de Taltal. Compilación, introducción y notas de Grete Mostny, tomos I-II. Fondo Histórico y Bibliográfico J. T. Medina, Santiago.

NIEMEYER, H. y V. SCHIAPPACASSE, 1963. Investigaciones arqueológicas en las terrazas de Conanoxa, valle de Camarones (provincia de Tarapacá). Revista Universitaria. Anales de la Universidad Católica de Chile. Anales de la Academia Chilena de Ciencias Naturales 26: 101-166.
NUÑEZ, L., Ms. La industria lítica del sitio Rinconada, Pampa del Tamarugal (provincia de Tarapacá).

NUÑEZ L., V. ZLATAR y P. NUÑEZ, 1972-1973. Reciente prospección de sitios arqueológicos componentes de un circuito transhumántico entre la costa y el borde occidental de Pampa del Tamarugal, norte de Chile. Separata de Etnia, 16-17: 1-6, 26-31. Museo Etnográfico Municipal D'Arce e Instituto de Investigaciones Antropológicas, Olavarría.

_ Ms. Excavaciones en el sitio Caleta Huelén-42.

NUÑEZ, L., C. MORAGAS y C. STAAL, Ms. Excavaciones en el sitio Tiliviche 1-b (provincia de Tarapacá).

NUÑEZ, L. y J. VARELA, 1966. Complejo preagrícola en el Salar de Huasco (provincia de Tarapacá). Revista Estudios Arqueológicos 2: 9-24.

NUÑEZ, P. y V. ZLATAR, Ms. Excavaciones en el sitio Aragón (provincia de Tarapacá).

TRUE, D. y L. NUÑEZ, Ms. Un piso habitacional temprano en el norte de Chile.

TRUE, D., L. NUÑEZ y P. NUÑEZ, 1970. Archaeological investigations in Northern Chile. Project Tarapaca. Preceramic resource. American Antiquity 35 (2): 170-184.

- 1971. Tarapaca-10: A workshop site in Northern Chile. Proceedings of the American Philosophical Society 115: 398-421. 